\title{
Integrated genomic and transcriptomic analysis revealed mutation patterns of de-differentiated liposarcoma and leiomyosarcoma
}

\author{
Wenshuai Liu ${ }^{1 \dagger}$, Hanxing Tong ${ }^{2 \dagger}$, Chenlu Zhang ${ }^{3+}$, Rongyuan Zhuang ${ }^{3}, \mathrm{He} \mathrm{Guo}^{4}$, Chentao Lv ${ }^{1}$, Hua Yang ${ }^{1}$, \\ Qiaowei Lin², Xi Guo ${ }^{3}$, Zhiming Wang ${ }^{3}$, Yan Wang ${ }^{3}$, Feng Shen ${ }^{3}$, Shengzhou Wang ${ }^{4}$, Chun Dai ${ }^{4}$, Guan Wang ${ }^{4}$, \\ Jun Liu', Weiqi Lu' ${ }^{2}$, Yong Zhang ${ }^{2^{*}}$ and Yuhong Zhou ${ }^{3^{*}}$ (D)
}

\begin{abstract}
Background: Treating patients with advanced sarcomas is challenging due to great histologic diversity among its subtypes. Leiomyosarcoma (LMS) and de-differentiated liposarcoma (DDLPS) are two common and aggressive subtypes of soft tissue sarcoma (STS). They differ significantly in histology and clinical behaviors. However, the molecular driving force behind the difference is unclear.

Methods: We collected 20 LMS and 12 DDLPS samples and performed whole exome sequencing (WES) to obtain their somatic mutation profiles. We also performed RNA-Seq to analyze the transcriptomes of 8 each of the LMS and DDLPS samples and obtained information about differential gene expression, pathway enrichment, immune cell infiltration in tumor microenvironment, and chromosomal rearrangement including gene fusions. Selected gene fusion events from the RNA-seq prediction were checked by RT-PCR in tandem with Sanger sequencing.

Results: We detected loss of function mutation and deletion of tumor suppressors mostly in LMS, and oncogene amplification mostly in DDLPS. A focal amplification affecting chromosome 12q13-15 region which encodes MDM2, CDK4 and HMGA2 is notable in DDLPS. Mutations in TP53, ATRX, PTEN, and RB1 are identified in LMS but not DDLPS, while mutation of HERC2 is only identified in DDLPS but not LMS. RNA-seq revealed overexpression of MDM2, CDK4 and HMGA2 in DDLPS and down-regulation of TP53 and RB1 in LMS. It also detected more fusion events in DDLPS than LMS (4.5 vs. 1, $p=0.0195$ ), and the ones involving chromosome 12 in DDLPS stand out. RT-PCR and Sanger sequencing verified the majority of the fusion events in DDLPS but only one event in LMS selected to be tested. The tumor microenvironmental signatures are highly correlated with histologic types. DDLPS has more endothelial cells and fibroblasts content than LMS.

Conclusions: Our analysis revealed different recurrent genetic variations in LMS and DDLPS including simultaneous upregulation of gene expression and gene copy number amplification of MDM2 and CDK4. Up-regulation of tumor related genes is favored in DDLPS, while loss of suppressor function is favored in LMS. DDLPS harbors more frequent fusion events which can generate neoepitopes and potentially targeted by personalized immune treatment.
\end{abstract}

Keywords: Soft tissue sarcoma, Gene fusion, Tumor microenvironment, Immune infiltration

\footnotetext{
* Correspondence: 13681971072@163.com; zhou.yuhong@zs-hospital.sh.cn

'Wenshuai Liu, Hanxing Tong and Chenlu Zhang contributed equally to this work.

${ }^{2}$ Department of General Surgery, Zhongshan Hospital, Fudan University,

Shanghai, China

${ }^{3}$ Department of Medical Oncology, Zhongshan Hospital, Fudan University,

Shanghai, China

Full list of author information is available at the end of the article
}

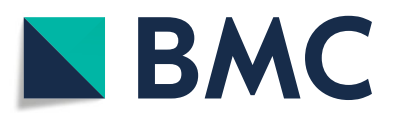

(- The Author(s). 2020 Open Access This article is licensed under a Creative Commons Attribution 4.0 International License, which permits use, sharing, adaptation, distribution and reproduction in any medium or format, as long as you give appropriate credit to the original author(s) and the source, provide a link to the Creative Commons licence, and indicate if changes were made. The images or other third party material in this article are included in the article's Creative Commons licence, unless indicated otherwise in a credit line to the material. If material is not included in the article's Creative Commons licence and your intended use is not permitted by statutory regulation or exceeds the permitted use, you will need to obtain permission directly from the copyright holder. To view a copy of this licence, visit http://creativecommons.org/licenses/by/4.0/. The Creative Commons Public Domain Dedication waiver (http://creativecommons.org/publicdomain/zero/1.0/) applies to the data made available in this article, unless otherwise stated in a credit line to the data. 


\section{Background}

Soft tissue sarcoma (STS) is a rare malignant tumor but has a great diversity. It occurs about 2.91 times per 100, 000 people and accounts for $1.05 \%$ of overall cancer incidents in China [1]. However, there are more that $50 \mathrm{rec}-$ ognized STS subtypes and that makes the accurate diagnosis and treatment a challenge [2]. LPS and LMS are among the most frequent STS subtypes, accounting for 5.75 and $5.97 \%$ of all cases respectively [1]. At the same time, LMS and a subtype of LPS, DDLPS, are the two most aggressive types, both having a complex karyotype and high recurrence rate [3]. Nevertheless, they differ significantly in histology, biology, and clinical behaviors [4]. For example, DDLPS tends to recur locally, while LMS has a higher chance of distant recurrence. It is unclear what genetic and molecular differences may have contributed to the distinction between LMS and DDLPS. In order to gain insight about their origins and molecular difference, and enlighten differential treatment, we performed integral WES and RNA-seq analysis and compared the two subtypes. We identified potential key mutational events that may have differentially driven tumorigenesis and discovered different tumor microenvironments in LMS and DDLPS. We also detected few neoantigens, mostly in DDLPS, which may be targeted by personalized immune therapy.

\section{Methods}

\section{Patients and specimens}

Twenty LMS and twelve DDLPS tissue samples and matched peripheral blood from the same patient were collected at Zhongshan Hospital of Fudan University with patient consent. The study protocol was reviewed and approved by the Ethics Committee of Zhongshan Hospital. H\&E stained histological sections were reviewed by an expert pathologist to confirm subtype diagnosis and tumor content to be above $20 \%$. The cytogenetic, immunohistochemistry (IHC), and pathologic lab data, as well as clinical treatment and outcome records were manually curated from the electronic medical records of the hospital.

\section{WES}

DNA in formalin-fixed, paraffin-embedded (FFPE) tissue samples was extracted using MagMAX FFPE DNA/RNA Ultra kit (cat\# A31881, ThermoFisher). DNA in snap-frozen tissue and peripheral whole blood was extracted using Maxwell RSC blood DNA kit (cat\# AS1400, Promega). Purified DNA was sheared with a Covaris L220 sonicator and hybridized to the probes in Agilent SureSelect XT Human All Exon V7 kit (cat\# 5991-9039, Agilent) for exome enrichment. Captured exome DNA was PCR amplified, end-repaired, and attached to the adapters and barcode using SureSelect XT HS and Low Input Library Preparation Kit for ILM (Pre
PCR) kit (cat\# G9704, Agilent, Santa Clara, CA, USA) according to the manufacturer's specification. The prepared libraries were sequenced on an Illumina NovaSeq-6000 Sequencing System to generate $150 \times 150$-bp paired-end reads. The image analysis and base calling were done using the Illumina onboard RTA3 program with default parameters. After removing adapters and low-quality reads, the reads were aligned to NCBI human genome reference assembly hg19 using the Burrows-Wheeler Aligner (BWA) alignment algorithm and further processed using the Genome Analysis Toolkit (GATK, version 3.5), including the GATK Realigner Target Creator to identify regions that needed to be realigned. Single-nucleotide variants (SNV), Indel, and copy number variation $(\mathrm{CNV})$ were determined using the MuTect/ANNOVAR/dbNSFP31, VarscanIndel, and CNVnator softwares respectively as reported in [5]. During the mutation calling, the reads from the tumor sample were compared with the paired blood from the same patient to generate a list of somatic mutations. The called somatic mutations were then filtered and annotated using Variant Effect Predictor (VEP) package (hg19 version) [6].

Mutation analysis and visualization were performed using the R (v3.3.3) (http://www.r-project.org), Bioconductor (v3.4) (http://www.Bioconductor.org), and MAFtools softwares $[7,8]$. Mutation data in maf format was generated using VCF2MAF v1.6.16 [9]. CNA of individual samples was determined using WES data by Control-FREEC software [10], with the window size set to $500 \mathrm{bp}$ and step size set to $250 \mathrm{bp}$ as recommended by the software authors. Recurrent focal and broad CNA in patient group were identified by GISTIC2.0 and GSEA $[11,12]$.

\section{Tumor mutational burden and microsatellite status}

Absolute counts of the tumor mutational burden (TMB) were calculated from the total number of nonsynonymous somatic mutations detected in WES using a published algorithm [13]. Autosomal microsatellite trains containing $1-5 \mathrm{bp}$ repeating subunits in length and comprising five or more repeats referenced to GRCh37/hg19 were identified using MISA (http://pgrc.ipk-gatersleben. de/misa/misa.html) [14]. Microsatellite Instability (MSI) score was defined as "number of unstable microsatellite sites / total valid sites" and a score $\geq 3.5 \%$ is regarded as high (MSI-High) and $<3.5 \%$ as low (MSI-Low) [15].

\section{RNA-Seq}

RNA was purified from FFPE tissue using the MagMAX FFPE DNA/RNA Ultra kit, reverse-transcribed using the NEBNext RNA First Strand Synthesis Module (cat\# E7525S, NEB) and NEBNext Ultra II non-directional RNA Second Strand Synthesis Module (cat\# E6111S, NEB) for cDNA Synthesis. RNA-seq libraries were prepared from the cDNA using SureSelect XT HS and 
Low Input Library Preparation Kit for ILM (Pre PCR) (cat\# G9704, Agilent, Santa Clara, CA, USA) following the manufacturer's instruction. The libraries were sequenced on an Illumina NovaSeq-6000 Sequencing System to generate $150 \times 150$ paired-end reads. Using FFPE sample for RNA-Seq is a more difficult task than using fresh or snap-frozen tissue. However, FFPE is still the most accessible tumor samples so studies have been lunched to test the use of RNA from FFPE sample for RNA-seq analysis and established reliable protocols [16, 17]. The protocols have since been adopted to routine use in oncogenomics studies and commercial kits have been developed by several suppliers, including the two New England Biolabs kits (NEBNext RNA first strand synthesis module and NEBNext Ultra II non-directional RNA second strand synthesis module) we used above.

\section{Differential gene expression analysis}

Reads from RNA-Seq were quality checked, filtered, and aligned to reference genome (hg19) using STAR (version 020201) and assembled using StringTie2 (version 1.3.5) $[18,19]$. A count matrix of raw reads was generated and the reads were further normalized by the upper quartile counts. A list of genes expressed differentially between LMS and DDLPS was compiled using DESeq2 [20]. The significance threshold was set at adjusted $P$-value $<0.05$ and $\log 2$ fold change $(\operatorname{LogFC})>1$.

\section{Pathway analysis}

Biological pathway analysis was done with the Kyoto Encyclopedia of Genes and Genomes (KEGG) pathway analysis and Gene Set Enrichment Analysis (GSEA) softwares using the above gene expression data and visualized by the NetworkAnalyst (http://www.networkanalyst.ca) software [12, 21, 22].

\section{Gene fusion analysis NGS sequencing and bioinformatic prediction}

STAR FUSION 1.8.0 was used to identify gene fusion events from the above mapped RNA-seq reads [23]. The standard setting, except a custom filter of at least 3 positive reads per event, was applied to increase calling stringency.

\section{Reverse transcription PCR (RT-PCR) and sanger sequencing confirmation}

Extracted RNA from tumor tissue was reverse transcribed using SuperScript Double Stranded cDNA synthesis kit (cat\#11917010, Invitrogen) and random hexamer oligos as the primer. The produced cDNA was amplified by PCR using a left primer complementary to the upper stream sequence and a right primer complementary to the downstream sequence across the fusion junction as predicted by the above NGS-STAR FUSION pipeline. The primer sequences, fusion junction sequences and reads number are listed in Supplementary Table S1. The PCR product was isolated by electrophoresis on agarose gel, extracted, and cloned in pCR4-TOPO vector (cat\#450030, ThermoFisher), then subjected to Sanger sequencing to verify the fusion event.

\section{Immune classification of STS}

The Microenvironment Cell Populations-counter (MCPcounter) method was used to profile tumor microenvironment infiltrating immune cells with RNA-seq derived Transcripts Per Million (TPM) matrix generated by the stringtie2 pipeline [24, 25]. Abundance scores for eight immune populations ( $\mathrm{T}$ cells, CD8+ $\mathrm{T}$ cells, cytotoxic lymphocytes, natural killer cells, B cell lineage, monocytic lineage, myeloid dendritic cells and neutrophils), and two stromal populations (endothelial cells and fibroblasts) are calculated using the signature composition defined previously [24]. Unsupervised clustering of samples was performed based on the metagene Z-score of MCP-counter.

\section{Statistical analysis}

Statistical analysis was performed with $\mathrm{R}$ (version 3.6.3) or SPSS (version 25). Unless stated otherwise, unpaired t-test was used in all group comparisons. A result of $p<$ 0.05 was considered significant.

\section{Results}

Genomic analysis has greatly expanded people's knowledge in the genetic composition of cancer mutations and in many cases helped design of new treatments. In the hope of using genomic information to guide the diagnosis and treatment of STS, we recruited thirty-two patients, twenty with LMS and twelve with DDLPS, at Zhongshan Hospital of Fudan University, Shanghai, China. The patient characteristics are indicated in Table 1.

\section{Somatic mutation profile}

Whole exome sequencing was run on both the tumor and paired peripheral blood (normal) samples from the same patient and compared to give somatic mutations in the tumor for each patient. Overall, the tumor mutational burden (TMB) of STS with a median of 2.27 counts/Mb is relatively low in comparison with other cancers [4]. There is no significant difference of TMB between LMS and DDLPS (median 2.61 and 1.97 counts $/ \mathrm{Mb}$ respectively, $p=0.105$ ) (Table 1). A large number of somatic mutations led by MUC16 (66\%) and PABPC3 (56\%) were detected (Supplementary Table S2). In order to focus on the 
Table 1 Summary of Clinical information and WES and RNA-seq analysis

\begin{tabular}{|c|c|c|c|}
\hline & All & LMS & DDLPS \\
\hline \multicolumn{4}{|l|}{ Sample size } \\
\hline WES & 32 & 20 & 12 \\
\hline RNA-seq & 16 & 8 & 8 \\
\hline \multicolumn{4}{|l|}{ Age (years) } \\
\hline Median & 51 & 49.5 & 54 \\
\hline Range & $16-70$ & $16-65$ & $37-70$ \\
\hline \multicolumn{4}{|l|}{ Gender (\%) } \\
\hline Male & $12(37.5 \%)$ & $3(15 \%)$ & $9(75 \%)$ \\
\hline Female & $20(62.5 \%)$ & 17 (85\%) & $3(25 \%)$ \\
\hline \multicolumn{4}{|l|}{ WES } \\
\hline \multicolumn{4}{|l|}{ TMB (counts/Mb) } \\
\hline Median & 2.27 & 2.61 & 1.97 \\
\hline Range & $1.20-16.1$ & $1.57-16.1$ & $1.20-4.51$ \\
\hline \multicolumn{4}{|l|}{ MSI score (\%) } \\
\hline High & 1 (3.1\%) & $1(5 \%)$ & $0(0 \%)$ \\
\hline Low & 31 (96.9\%) & 19 (95\%) & $12(100 \%)$ \\
\hline SNP + Indel & 2887 & 2110 & 1088 \\
\hline CNA gain & & 113 & 1089 \\
\hline \multicolumn{4}{|l|}{ RNA-seq } \\
\hline Up-regulated & & 2404 & 2364 \\
\hline Gene fusion & 40 & 4 & 36 \\
\hline RT-PCR verified & 8/12 (66.7\%) & $1 / 4(25 \%)$ & $7 / 8(87.5 \%)$ \\
\hline
\end{tabular}

* TMB is reported as counts/Mb. It is calculated by all nonsynonymous mutations detected in WES divided by $35 \mathrm{Mb}$. $35 \mathrm{Mb}$ is the sum of exome probes in WES

WES whole exome sequencing, TMB tumor mutation burden, MSI microsatellite instability

genes with a known function in cancer or reported to related to cancer, we filtered the mutations against a list of 1190 member cancer related genes compiled from literature and OncoKB (http:|crwww.oncokb.org) [26]. There is a distinct distribution of somatic mutations between LMS and DDLPS among the top 30 most mutated cancer-related genes (Fig. 1). Eleven out of the 20 (55\%) LMS patients had mutation in TP53. In contrary, none of the DDLPS patient showed TP53 mutation. Similarly, FLG is mutated in 9 (45\%) LMS patients but only in 1 (8.3\%) DDLPS patient. ANHAK, ATRX, CSPG4 and PCMTD1 each was mutated in 5 (25\%) LMS patients and none DDLPS patients. HERC2 was mutated in 5/8 (41.7\%) DDLPS patients, C12orf55, DNAJC16, PTPRQ, and TIAM1 each was mutated in 3 (25\%) DDLPS patients. None of the above five genes had mutation in LMS patients (Fig. 1). As a comparison, the mutation frequency of HERC2 in TCGA-SARC dataset was 11/58 (19.0\%) for DDLPS and 18/102 (17.6\%) for LMS cases. The mutation frequency of TP53 in TCGASARC dataset was 10/58 (17.2\%) for DDLPS and 80/ $102(78.4 \%)$ for LMS cases. Therefore HERC2 mutation is imbalanced and TP53 mutation is generally lower in both DDLPS and LMS groups in our patients as comparing to that of TCGA-SARC dataset [4]. This may arise from the difference of patient composition. All 32 cases in our group are ethnic Chinese but only 2/160 (1.25\%) are Asian and 140/ $160(87.5 \%)$ are white in the LMS and DDLPS cases of TCGA-SARC dataset. The number of cases is small in both groups though and that prevented us to draw a definitive answer about the distribution difference. The absence of HERC2 mutation and biased presence of TP53 mutation in the LMS group is an interesting observation. HERC2 belongs to E3 ubiquitin protein ligases, and can modulate p53 activity through regulating p53 oligomerization independent of MDM2 [27]. Probably because the mutations that affect p53 tetramerization disrupt the HERC2-p53 interaction, therefore HERC2 mutations are redundant in LMS with mutant TP53.

\section{SCNA in LMS and DDLPS}

In addition to SNV and indel, gene amplification and deletion are also important contributors to carcinogenesis. To further reveal the somatic copy number alterations (SCNA) in Chinese sarcoma patients, we used GIST IC2.0 to detect SCNA (Supplementary Tables S3-5). We found significant chromosomal loss in LMS peaked at cytobands 10q22.1, 13q34, and 17p13.1, where PTEN, RB1 and TP53 are located respectively (Fig. 2a). All these genes are important tumor suppressors. This suggested that chromosomal loss affecting tumor suppressor genes is a hallmark of LMS. On the other hand, we found a focal amplification peaked at 12q14.1, included in its wide peak are MDM2 (12q15), CDK4 (12q14.1) and HMGA2 (12q14.3) (Fig. 2b). We further verified the prediction of GISTIC by GSEA analysis under the positional mode. We used chromosomal neighbors as the input gene set instead of signaling pathway members in GSEA and found 12q14-12q15 regions are indeed enriched in DDLPS (EnrichmentScore ES $=0.662$ for $12 \mathrm{q} 14$ and ES = 0.784 for $12 \mathrm{q} 15$ ) (Fig. 2c and data not shown). MDM2 and CDK4 are both important cell cycle regulating genes. HMGA2 is a chromosomal structure organization protein and may also play a role as a transcription factor. It has been reported that alteration of HMGA2 is associated with myxoid liposarcoma and takes part in adipogenesis and mesenchymal differentiation [28, 29]. Amplification of $12 \mathrm{q} 14$ and $12 \mathrm{q} 15$ regions likely upregulated the above genes important in regulation of cell cycle and transformation of adipocytic tissue, therefore drove DDLPS. At the gene level, we detected co- 


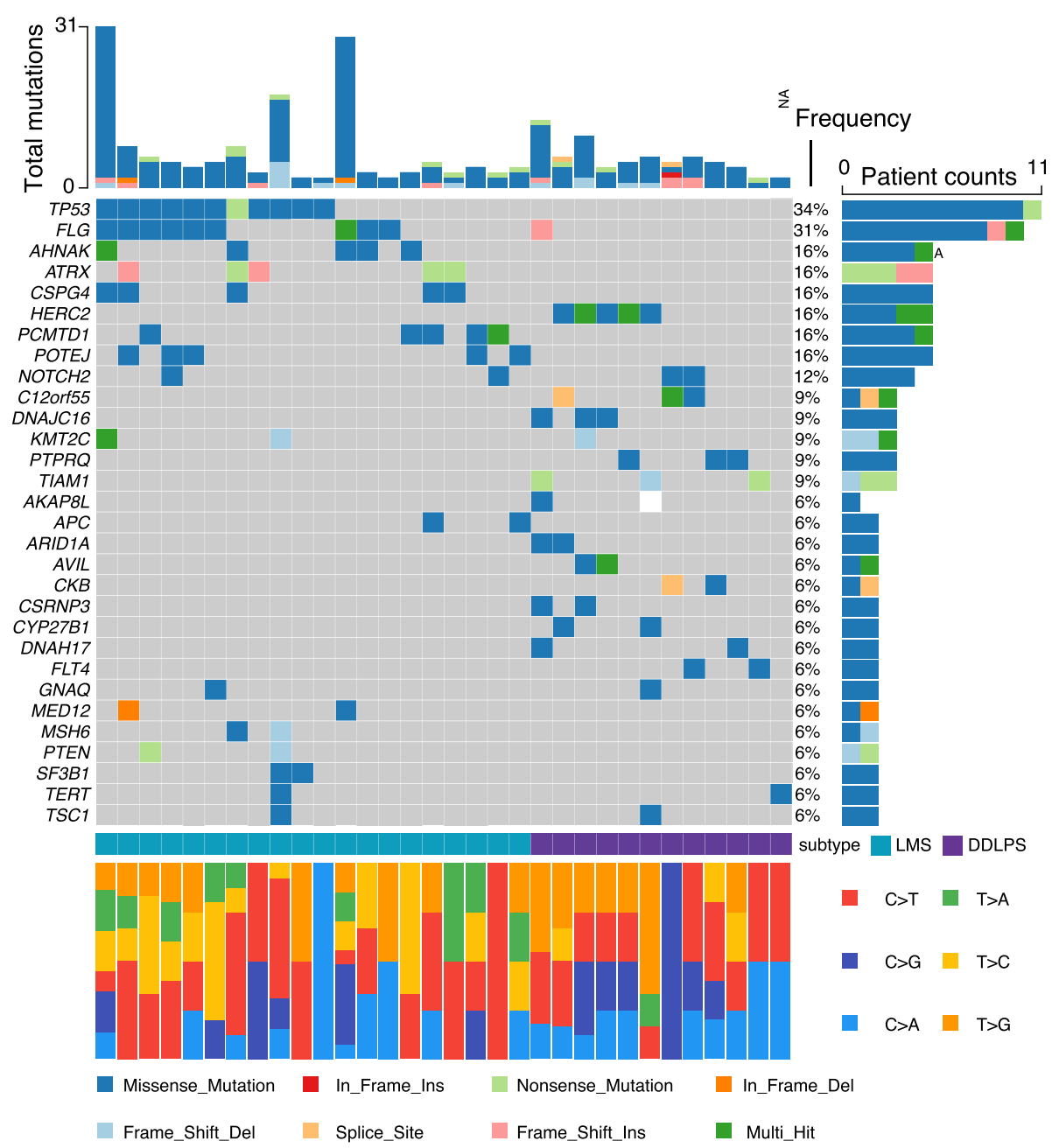

Fig. 1 Top30 frequently mutated genes in LMS and DDLPS. Patients are grouped by disease (Cerulean: LMS; Purple: DDLPS) and the genes are ranked by their mutation frequencies. The top chart indicates total number of mutations in each patient. The types of mutation and nucleotide transition are color coded

amplification of $M D M 2$ and CDK4 in more than $90 \%$ DDLPS cases. Such co-amplification combined with TP53 inactivation would result in cell proliferation, and is very likely the initiating events to drive fat tumorigenesis in DDLPS [30].

Additional genes detected by GISTIC 2.0 with amplification and deletion are listed in supplement Table S2-S5. In LMS, 96 genes are significantly amplified, while as many as 4532 genes are significantly deleted (confidence level 0.95), indicating that deletion is much more frequent than amplification. In DDLPS 1089 genes are amplified and no genes are significantly deleted. These observations are consistent with previous publications as reviewed in [3]. In DDLPS amplification of oncogenes such as MDM2, CDK4, HMGA2 and JUN are common, while in LMS deletion of tumor suppressor such as TP53, RB1, and
PTEN are preferred, indicating different patterns of CNA in DDLPS and LMS.

\section{Concurrent and exclusive mutations}

Given the possibility that synergistic or anti-synergistic interactions between genes may contribute to the origin or progression of LMS and DDLPS, we tested interactions among the somatic mutations called in WES analysis using the somaticInteractions function in MAFtools. (Fig. 3, and the full list in Supplementary Table S2. TP53 is mutually exclusive with BRD9 $(p<0.1)$ but co-occurs with Filaggrin $(F L G)(p<0.1)$. BRD9 is a subunit of the human BAF (SWI/SNF) nucleosome remodeling complex and has emerged as an attractive therapeutic target in cancer [31]. It has a bromodomain highly homologous to the bromodomain of BRD7, which is reported to interact with p53 and required for p53 

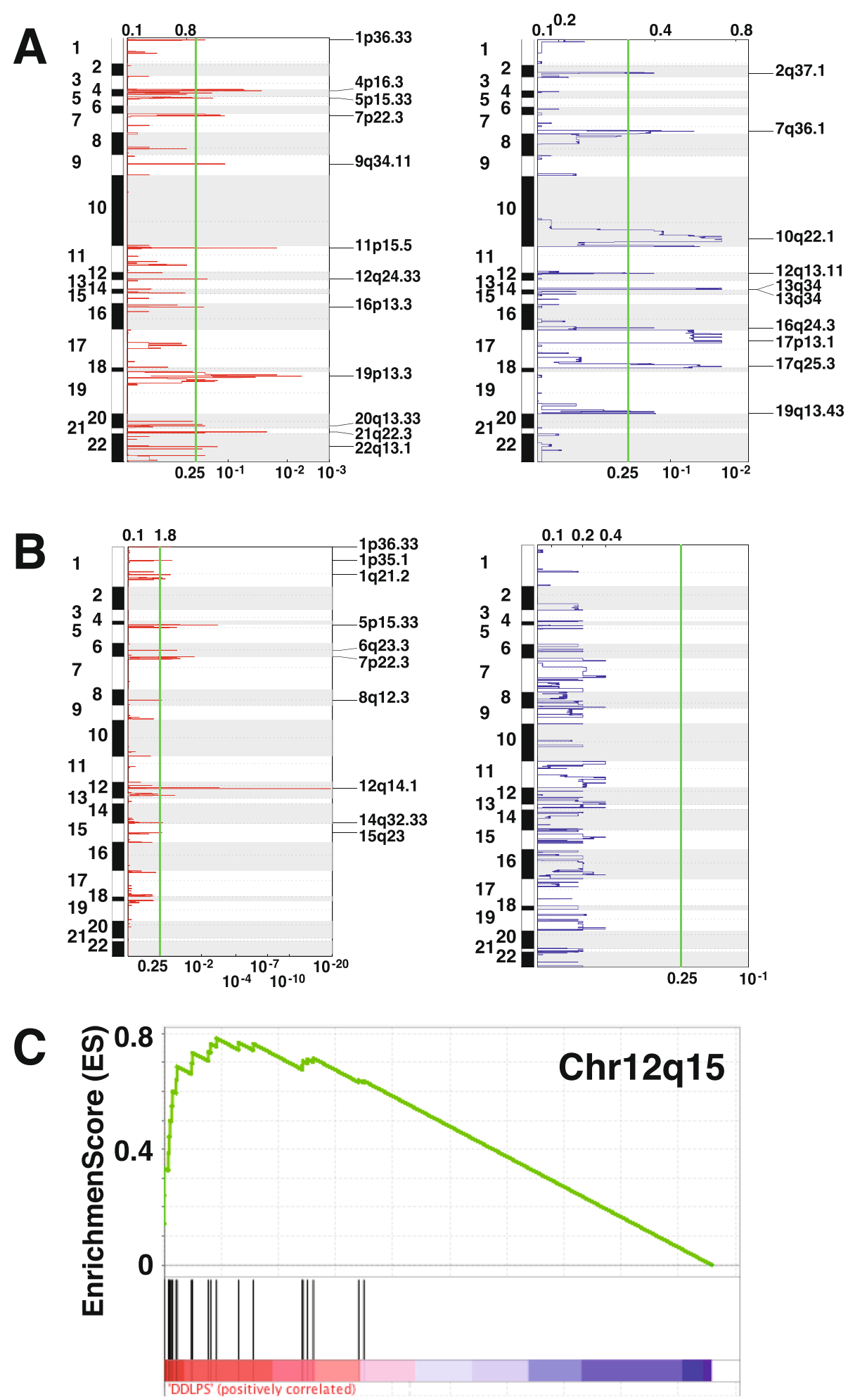

Fig. 2 Local gene copy number alteration.a-b GISTIC analysis of recurrent amplification (left, red) and deletion (right, blue) in LMS (a) and DDLPS (b). The y-axes represent genomic position of altered regions (left axis: chromosome; right axis: cytoband) and the $x$-axes represent normalized amplification signal (top axis) and significance by $Q$ value (bottom axis). The vertical green line represents the significance cutoff at $Q$ value $=0.25$. The most prominent amplification is seen around 12q14 in DDLPS. c GSEA by position. Gene sets of chromosomal neighbors are used as the input in GSEA which gives an ES =0.784 (nominal $p$-value 0.0177, FDR q-value 0.447) at Chr12q15. MDM2 is located at the junction of Chr12q14 and Chr12q15 and on the side of Chrq15 


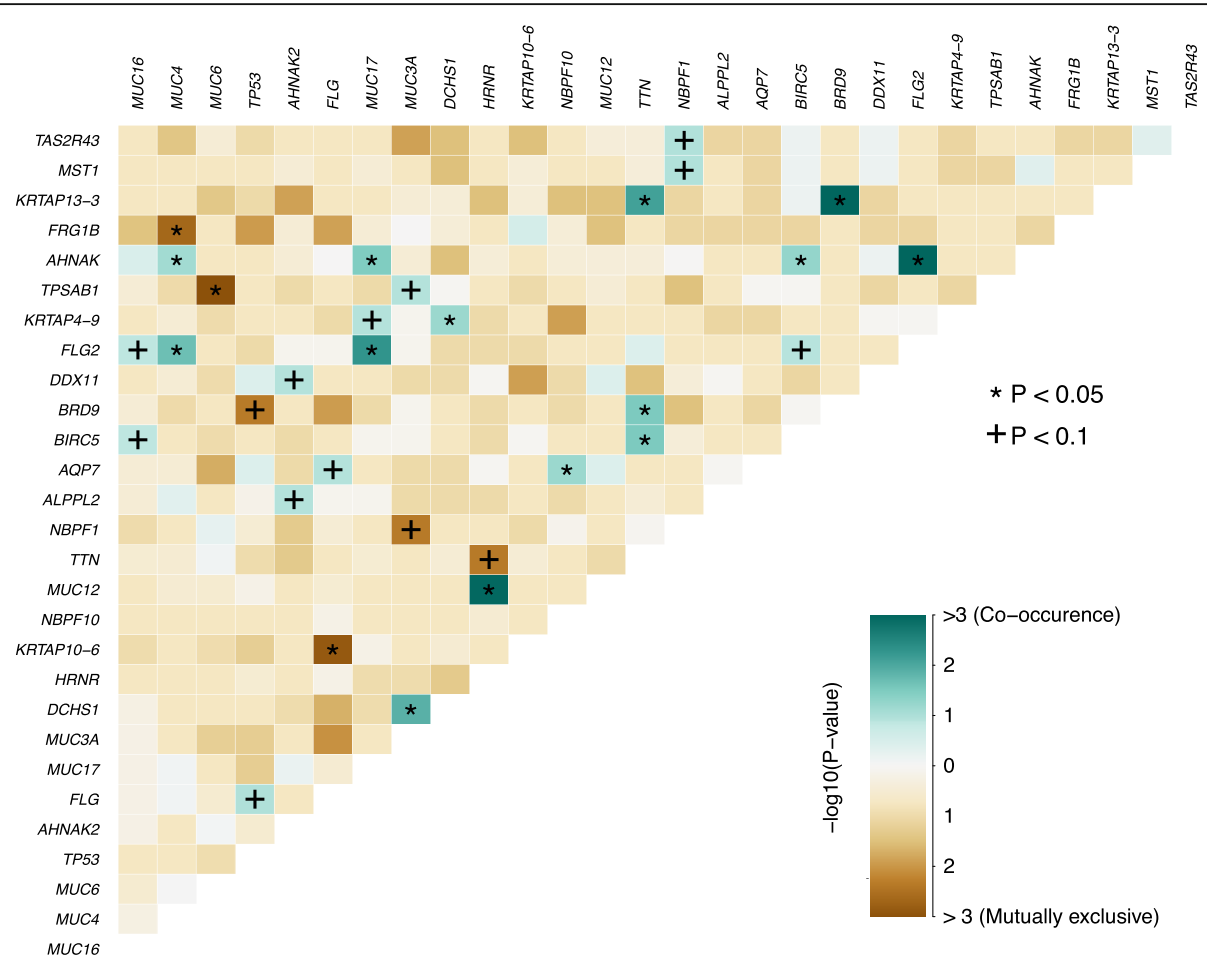

Fig. 3 Heatmap of predicted gene interactions in the 32 STS. The color scale represents - $\log 10 p$-value. The cadet blue indicates co-occurrence and the copper indicates mutual exclusion. Significant $p$-values are indicated by symbols. ${ }^{*}: p<0.05 ;+: p<0.1$

function [32]. Whether mutation of BRD9 promotes LMS through impairment of the p53 pathway is to be determined. FLG is a highly mutated cancer driver gene. Its mutation is also found in several other cancer types such as non-melanoma skin cancer, head and neck cancer, lung cancer, colorectal cancer, uterine cancer, and prostate cancer [33]. There has no reported synergic interaction between FLG and TP53 mutations in cancer yet, including STS. The above somatic interaction analysis may provide hints for exploring genes with unspecified functions in STS.

\section{RNA-seq revealed genes with differential expression}

Eight each of the LMS and DDLPS samples that had sufficient RNA quality were further subjected to RNA-Seq analysis. Although fresh or snap-frozen tissue are generally preferred over FFPE samples for RNA extraction and sequencing analysis, FFPE samples are by far the most accessible tissue samples. Dedicated works have been done both by academic labs and commercial manufacturers to develop special protocols to perform RNA-seq from FFPE samples. They have succeeded in using exome capturing for partially degraded RNA. We used a New England Biolabs protocol and companion kits in sample preparation for RNA-seq (see Methods) which has been proven functional and are widely used in NGS work with clinical samples [16, 17].

In total we identified 2396 genes expressed with significantly different levels in the LMS and DDLPS (Fig. 4 and Supplementary Table S6). Unsupervised clustering was performed to examine the discriminant effect of these genes. We found that all the LMS naturally clustered together, and so did the DDLPS samples. Ranked the highest in differential expression is $M D M 2$, with $\log \mathrm{FC}=4.12$ (DDLPS over LMS) and adjusted $P$-value $=1.73 \mathrm{e}-52$, consistent with previous studies [4, 34]. MDM2 is a proto-oncogene. It encodes a nuclear-localized E3 ubiquitin ligase and plays an important role in cell cycle regulation. Its copy number gain has been implicated in cancers including DDLPS [35, 36]. We also observed the gene copy number of $M D M 2$ is correlated with its mRNA in DDLPS (Fig. 5a). Similarly, another key cell cycle regulator $C D K 4$ is both highly upregulated in expression $(\log F C=3.61$, adjusted $p$-value $=1.09 \mathrm{e}-20)$ and amplified in gene copy number (Fig. 5a). These results suggest that hyper activation of cell cycle is highly correlated with and may possibly a driving force underlying DDLPS. This notion is supported by a recent study in human mesenchymal stem cell model in which co-expression of MDM2 and CDK4 


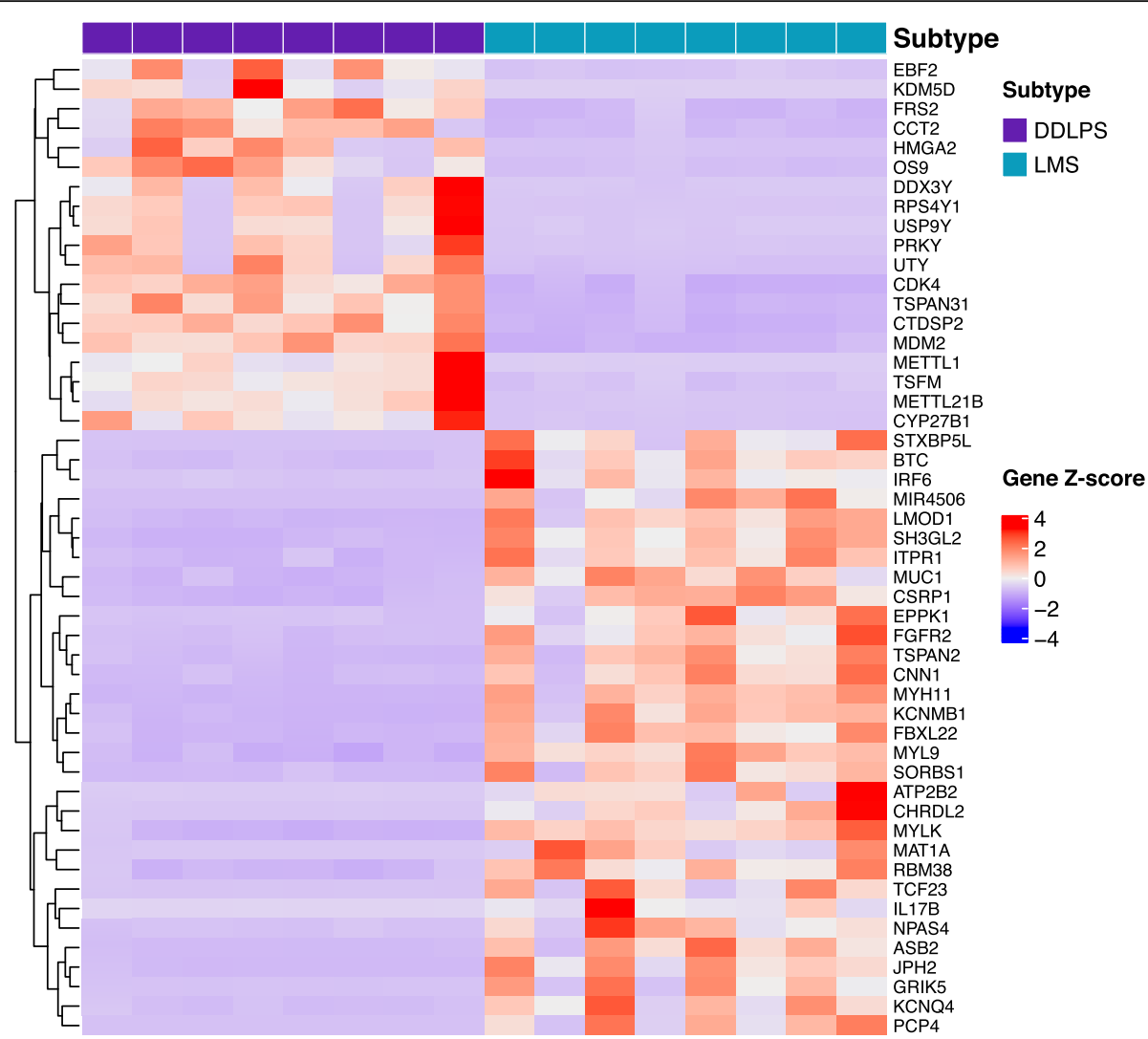

Fig. 4 Heatmap of top 50 differentially expressed genes between LMS (cerulean) and DDLPS (purple) with unsupervised clustering. One column represents one sample. The heat scale represents Z-score normalized gene expression. The red indicates up-regulated and the blue indicates down-regulated genes in DDLPS compared with LMS

produced DDLPS-like morphology [37]. Additionally, we found $J U N$ upregulation $(\operatorname{logFC}=1.45$, adjusted $P$ value $=0.0396$ ) in DDLPS (Supplementary Table S6). $J U N$ is a known player in blocking adipocytic differentiation so may have assisted tumorigenesis in adipose tissue.

Since we detected both CNA in WES and differential gene expression in RNA-seq, it would be of interest to see whether CNA is correlated with gene expression. Although the top varied genes such as MDM2 and CDK2 indeed have a good correlation between their DNA and mRNA level (Fig. 5a), the bulk of genes do not show this relationship, especially in the case of LMS (Fig. 5b-c). This may due to several factors. Gene expression is a tightly regulated cellular process. The copy number of available template DNA, especially for those which only have a moderate increase as the bulk of genes are, is only a small contributor of gene expression activity. The more important regulation can be attributed to the accessibility of the chromosomal region, and activities of promoters, specific transcription factors, and DNA dependent RNA polymerase machinery, and mRNA stability.
Pathway analysis of genes with differential expression To investigate the pathways affected in LMS and DDLPS, we performed Gene Set Enrichment Analysis (GSEA) (Table 2 and Supplementary Table S7) [12, 21]. We found several pathways are enriched in DDLPS $(E S>0)$ while depleted in LMS $(\mathrm{ES}<0)$. The pathways enriched in DDLPS include "Ubiquitin mediated proteolysis" $(\mathrm{ES}=0.472$, adjusted $p$-value $=0.002$ ) which has $M D M 2$ as a member. The pathways apparently enriched in LMS such as "Calcium signaling pathway", "Vascular smooth muscle contraction", and "Linoleic acid metabolism" (ES $=-0.37$, 0.44 , and -0.60 respectively, and adjusted $P$-values $=0.002$ for all) are probably due to the difference of the tissue origins rather than tumorigenesis $[38,39]$. Another notable pathway is spliceosome $(\mathrm{ES}=0.53$, adjusted $p$-value $=$ 0.003 ) because alteration in transcript splicing may result in different sets of antigens differentially recognizable by immune cells in different tumor environments.

\section{Distinct gene fusion patterns between DDLPS and LMS}

In the RNA-seq analysis, we identified in 3 (out of 8) DDLPS patients 4 potential gene fusion transcripts and 

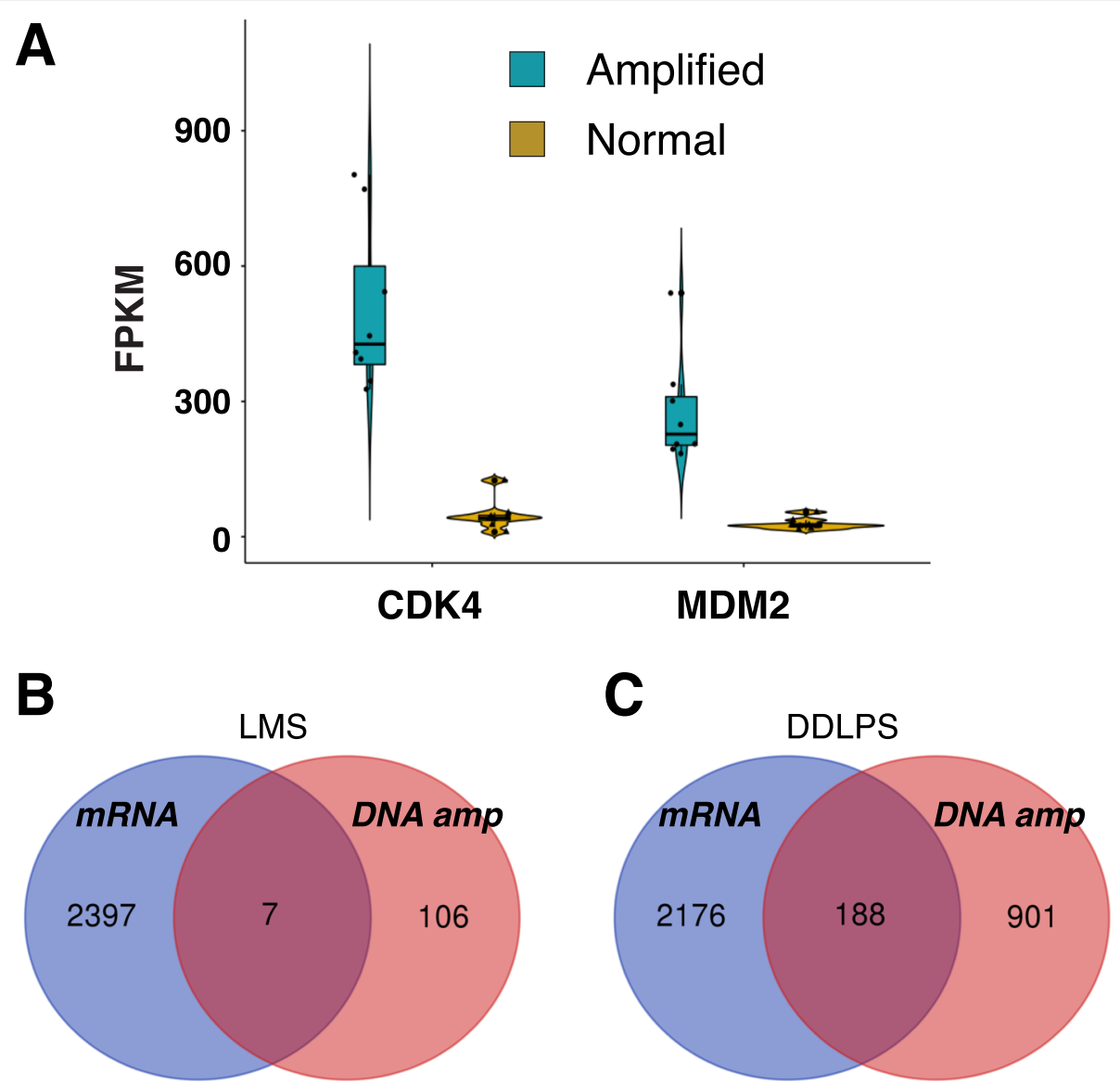

Fig. 5 Correlation of gene amplification detected in WES with transcripts upregulation detected in RNA-seq. a Violin-box plot comparing the expression of CDK4 (left) and MDM2 (right) in patients with (cerulean) and without (gold) gene amplification. The y-axis represents normalized reads number in FPKM detected in RNA-seq. The median value is indicated by the line inside the box. The width of the color shade indicates the distribution frequency. b-c Venn diagrams of detected genes amplified in WES (pink) and upregulated in expression in RNA-seq (blue) in LMS (b) and DDLPS (c) respectively

in all 8 LMS patients 36 fusion transcripts in total (Fig. 6 a-c and Supplementary Table S1). The distribution of fusion events is biased towards DDLPS over LMS $(p=$ 0.0195, Fig. 6c). There is no recurrent fusion transcript identified probably due to the small sample size. Fusion transcripts involving chromosome 12 are only found in DDLPS, including both inter- and intra-chromosomal rearrangements (Fig. 6b). MDM2 and RAB3IP are the most common fusion partners, and both are located in chromosome 12. There is also significant correlation

Table 2 Pathways enriched in GSEA (LMS in relative to DDLPS)

\begin{tabular}{|c|c|c|c|c|c|}
\hline Name & Total & Hits & EnrichmentScore & $p$-val & p-adj \\
\hline Calcium signaling pathway & 68 & 58 & -0.37 & 0.00017 & 0.0028 \\
\hline Vascular smooth muscle contraction & 30 & 22 & -0.44 & 0.000017 & 0.0028 \\
\hline Linoleic acid metabolism & 30 & 26 & -0.60 & 0.00018 & 0.0028 \\
\hline Ascorbate and aldarate metabolism & 34 & 28 & -0.67 & 0.00018 & 0.0028 \\
\hline Chronic myeloid leukemia & 33 & 27 & 0.53 & 0.000022 & 0.0028 \\
\hline p53 signaling pathway & 31 & 27 & 0.50 & 0.000022 & 0.0028 \\
\hline Spliceosome & 27 & 22 & 0.52 & 0.000022 & 0.0028 \\
\hline AGE-RAGE signaling pathway in diabetic complications & 18 & 16 & 0.47 & 0.000023 & 0.0028 \\
\hline Ubiquitin mediated proteolysis & 27 & 25 & 0.47 & 0.000023 & 0.0028 \\
\hline Osteoclast differentiation & 44 & 39 & 0.50 & 0.000023 & 0.0028 \\
\hline
\end{tabular}




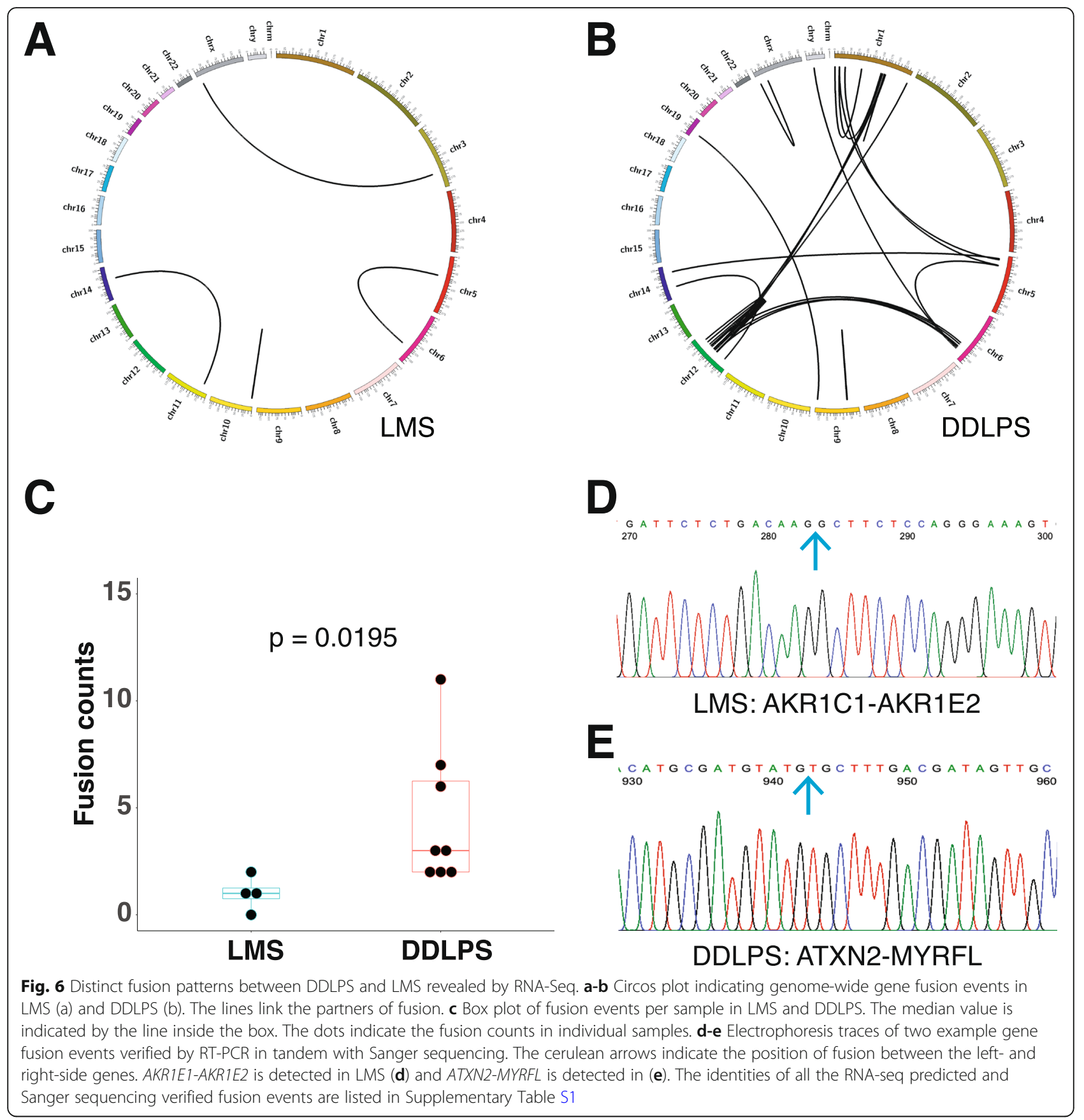

between $M D M 2 / C D K 4$ amplification and chromosome 12 rearrangement $(P<0.001$, Fig. $7 \mathrm{~b})$. Gene fusions can occur as a result of chromosomal rearrangements such as translocation, interstitial deletion, or inversion during DNA replication, which are more common in DDLPS than in LMS (Fig. 6 a-b). Therefore, it is not a surprise to see gene fusions more frequently in DDPLS, especially in chromosome 12 where ring or giant marker chromosomes often occur $[4,40]$. Peptides generated from the identified fusion transcripts may be a potential source of tumor neoantigens that can be targeted to produce safer and patient specific CAR-T cells for immunotherapy [41]. Although RNA-seq by NGS method is good at high throughput survey of all possible gene fusion events in the sample, it relies on bioinformatic models to predict the fusion. This may potentially introduce errors. We selected all 4 predicted gene fusion transcripts in LMS and 8 out of 36 in DDLPS to verify with RT-PCR in tandem with Sanger sequencing. We were able to verify only 1 event in LMS but 7 out of 8 events in DDLPS (Fig. $6 \mathrm{~d}$ and 


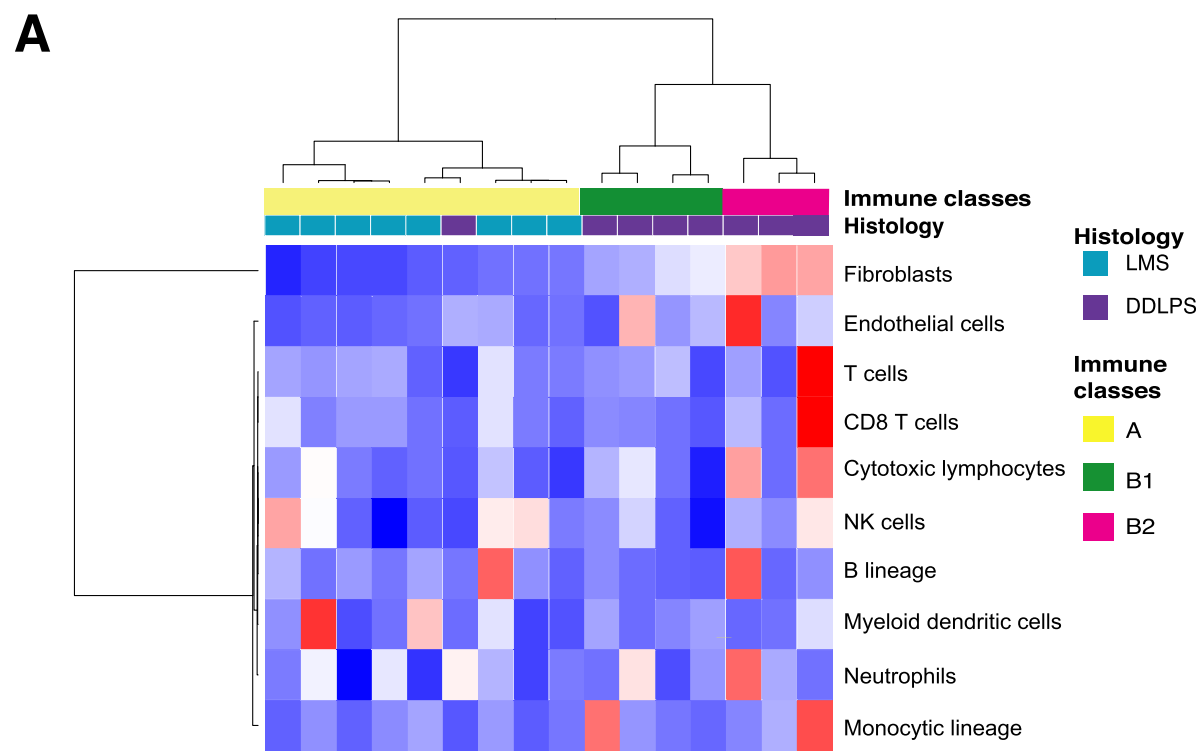

B
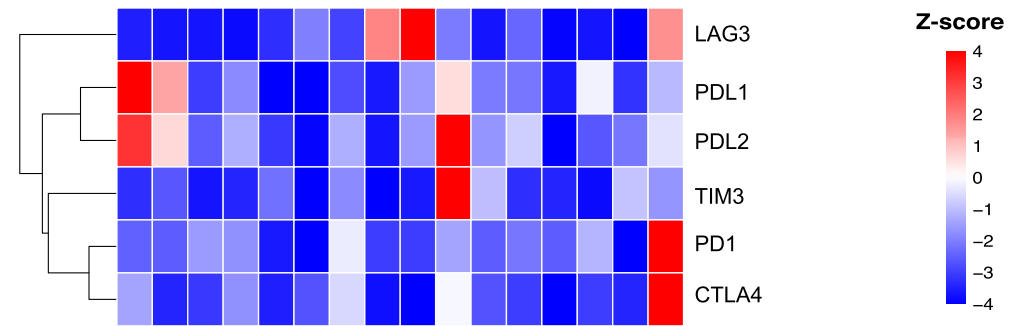

Fig. 7 Stromal and immune cell infiltration in tumor microenvironment. a Unsupervised clustering of LMS (cerulean) and DDLPS (purple) samples by MCP-counter Z-scores. $\mathbf{b}$ Expression of genes related to immune checkpoints. Each column represents one patient and is aligned between (a) and (b)

Supplementary Table S1). This confirmed that gene fusions are relatively more frequent in DDLPS but scarce in LMS and further highlighted that neoantigen based immune therapy may have a higher success rate in DDLPS than in LMS.

\section{LMS and DDLPS have different profiles of tumor infiltrating immune cells}

The status of tumor microenvironment (TME) is an important factor when immune therapy is considered. Previous studies revealed that STS immune subtypes are associated with response rate to PD1 blockade. To access the TME in LMS and DDLPS and compare the difference between them, we used MCP-counter to profile tumor infiltrating immune cells in the two subtypes following a recent study in STS (Fig. 7) [24]. Among the eight immune populations ( $\mathrm{T}$ cells, $\mathrm{CD} 8+\mathrm{T}$ cells, cytotoxic lymphocytes, natural killer cells, B cell lineage, monocytic lineage, myeloid dendritic cells and neutrophils) and two stromal populations (endothelial cells and fibroblasts) used by MCP-counter to categorize sarcomas immune classes (SIC), DDLPS has a higher signature score than LMS in both the non-immune cell populations $(p=0.002$ for fibroblasts and $p=0.047$ for endothelial cells). Increased endothelial cell signature score has been shown to associate with a high density of CD34+ endothelial cells and enhancer free endothelial-driven angiogenesis in STS [4]. The high fibroblasts signature score of DDLPS is consistent with its mesenchymal origin. The previous TCGA study suggested that CD8 $+\mathrm{T}$ cells are higher in DDLPS than in LMS $(P<0.01)$, but no such significance was observed in the current study $(P=0.76)$ [4].

Unsupervised clustering of the 16 DDLPS/LMS RNA samples according to their MCP-counter Z-scores revealed a bipartite pattern (Fig. 7a). All the LMS samples were classified to SIC A as defined by Petitprez et al., while most DDLPS samples except one were classified to SIC B [24]. The SIC B cluster can be further divided to two subclasses (B1 and B2) with the B2 subclass has a higher immune cell content in general. We also looked the expression of individual immune checkpoint molecules in the sample. It showed a higher representation of PD-L1 in LMS and higher TIM3, PD1 and CTLA4 in DDLPS in some individual samples. Overall, the immune checkpoint point molecules are not highly active consistent to the observed ICI treatment efficiency in STS. 
However, the expression profiles of individual patients are worth to check when an ICI prescription is considered.

\section{Discussion}

Our analysis revealed distinct mutation patterns and tumor microenvironments between LMS and DDLPS. This further testifies the diversity of STS and highlights the necessity of differential diagnosis and treatment of STS.

Genetic changes drive phenotypic change and eventually clinical manifestation and outcomes of disease. Both LMS and DDLPS belong to complex-karyotype STSs with an unbalanced karyotype and severe genomic aberrations. In this study, we revealed multiple chromosomal rearrangements in DDLPS, particularly the ones involving Chr12. These rearrangements were correlated with abundant gene amplification and fusion events as well. In contrary, LMS has fewer chromosomal level rearrangements, gene amplification and gene fusions (Figs. 3, 5 , and 6). Notably high gene copy amplification and expression of MDM2 and CDK4 are detected. The two genes have been reported to promote the transformation of mesenchymal cells to DDLPS and it was suggested that they work together in the process [30, 35-37]. A simultaneous detection of highly upregulated gene expression upregulation and gene copy number of both genes in this report further strengths their role in DDLP $\mathrm{S}$ tumorigenesis. Therapies targeting to MDM2/CDK4 axis is expected to relief patients from DDLPS.

A higher degree of genetic scrambling as seen in the DDLPS predicts a better response to immune therapies, for example, the checkpoint inhibition. This notion is supported by several Phase II studies of immune checkpoint inhibition (ICI) therapies in sarcoma, including SARC028, Alliance A091401 and PEMBROSARC [42-44]. Results from these studies revealed that although LMS has a higher TMB than DDLPS, the response rate of ICIs in LMS is lower than in DDLPS. For both LMS and DDLPS, the response rate of ICIs in an unselected population is low, and TME immunological landscape profiling can assist in identification of patients who are likely to respond to immunotherapies. Using MCP-counter and RNA-seq results, we simultaneously quantified multiple cell populations and focused on the immune cells. Results from unsupervised clustering of MCPcounter Z-scores partitioned LMS and DDLPS in two distinct immune classes. All LMS coalesced to class A which is low of immune cell infiltration, fibroblasts and endothelial cells. The majority of DDLPS samples clustered to class B which has higher fibroblasts, endothelial cells and immune cell infiltration. Tumors in class A are generally considered "cold" with a low response rate to ICIs $[45,46]$. For tumors in class B, two subgroups can be seen based on immune cell infiltrations. Class B1 has a relatively higher fibroblast and endothelial cells, and lower immune cell infiltration than class B2. In colon cancer, researchers have reported that immune and stromal classification was associated with molecular subtypes and patient's prognosis [47]. Although we can correlate the histology, mutational profile and immune classification of LMS and DDLPS with the good or poor patient prognosis, dedicated prospective trials evaluating chemotherapy, targeted therapy, or immunotherapy would elucidate better the role of immunological landscape profiling in STS treatment. The integrated WES and RNA-seq analysis derived from the current study can potentially contribute to developing new biomarkers for patient screening and prognosis prediction.

At the molecular level, it is notable that prevailing cancer related mutations, including TP53, AHNAK, and $A T R X$, are exclusively in LMS and absent in DDLPS. Conversely, HERC2 mutation only appears in DDLPS. These observations argue for that the two STS subtypes are derived from distinct cell origins and progressed through different mutational pathways. Diagnosis assisted by molecular profiling would bring additional value to patients besides pathological and histological approaches, especially when targeted or immune therapies are considered.

In spite of the above findings, several limitations remain in this study. Firstly, a relatively small number of patients with each histologic subtype were examined. This has prevented us to draw a clear-cut conclusion except for the few high frequency events we discussed above. STS is a rare disease, and it is even rarer for the individual subtypes. Therefore, analysis of few cases is still meaningful and has great reference value before a large number of cases can be available. Secondly, the evaluation of tumor microenvironment was based on RNA-seq of bulk cells. Although newer technologies such as single cell transcriptome analysis is available, due to the retrospective nature of this study, we could not obtain tissue specifically preserved and qualified for the single cell analysis. It also made it unlikely to verify the correlation of the molecular properties of the tumor with treatment in clinics. At last, we could not conduct survival analysis in the current cohort due to the relatively short follow-up time of the patients. These issues will be addressed in future studies and the insights we gained in the current study will help us to make appropriate patient stratification.

\section{Conclusion}

In conclusion LMS and DDPLS are distinct diseases as supported by our analysis of mutation pattern, gross genomic stability, and tumor infiltrating immune cell profiles. Chromosomal rearrangement may result in 
gene amplification and fusion, which are displayed the most obviously in Chr12 in DDLPS. These observations are consistent to the altered gene expression patterns in the two types of sarcoma. Neoantigens produced by gene fusion may open up new avenues for personalized immunotherapy in STS.

\section{Supplementary information}

Supplementary information accompanies this paper at https://doi.org/10. 1186/s12885-020-07456-2.

Additional file 1: Supplementary Table S1. list of gene fusion events and their sequences, and the oligos used and products detected by RTPCR.

Additional file 2: Supplementary Table S2. SNV list

Additional file 3: Supplementary Table S3. LMS_amplified_gene list in 0.95 confidence level

Additional file 4: Supplementary Table S4. LMS_deleted_gene list in 0.95 confidence level

Additional file 5:. Supplementary Table S5. DDLPS_ amplified_gene list in 0.95 confidence level

Additional file 6: Supplementary Table S6. RNA expression from RNA-seq analysis

Additional file 7: Supplementary Table S7. Pathways enriched from RNA-seq analysis

\section{Abbreviations}

STS: Soft-tissue sarcoma; WES: Whole exome sequencing; LPS: Liposarcoma; DDLPS: De-differentiated LPS; LMS: Leiomyosarcoma; CAR-T: Chimeric Antigen Receptor T; SNV: Single nucleotide variations; SCNA: Somatic copynumber alteration; CNA: Copy-number alteration; GATK: Genome Analysis Toolkits; CIN: Chromosomal instability; MSI: Microsatellite Instability; TPM: Transcripts Per Million; KEGG: Encyclopedia of Genes and Genomes; GSEA: Gene Set Enrichment Analysis; TMB: Tumor mutation burden; ICl: Immune checkpoint inhibitor

\section{Acknowledgements}

We thank all patients who participated in this study. We also thank our colleagues at GenomiCare Biotechnology for providing supports in sample preparation, sequencing and bioinformatic analysis.

\section{Authors' contributions}

WSL, HXT, CLZ: study design; RYZ and LCT: sample collection; JL and HG: statistical analysis and manuscript writing; CD: molecular genetic studies; GW: sequence alignment; $H Y, Q W L, X G, Z M W$ : clinical information management; YW, FS, WQL, JL: contributed to the interpretation of the results; YHZ, YZ: design, supervision, and manuscript writing. All authors read and approved the final manuscript.

\section{Funding}

This study was funded by Shanghai Municipal Health Commission Scientific Research Project, Grant Number: 20194Y0459. The funder did not participate in the study design nor data interpretation.

\section{Availability of data and materials}

The data analyzed in this article is available from the corresponding author on reasonable request.

\section{Ethics approval and consent to participate}

Written informed consents were obtained from all individual participants included in the study. All procedures performed involving human participants were in accordance with the ethical standards of Zhongshan Hospital, Fudan University and/or national research ethics committee and with the 1964 Helsinki declaration and its later amendments or comparable ethical standards. The study design was approved by the The Ethics Committee of Zhongshan Hospital, Fudan University (Approval no. B2012-
022). The ethics committee members include Jia Fan, Xinyu Qin, Weixin Niu, Jiyao Wang, Kangda Liu, Haiqing Li, Huizhu Heng, Xuening Li, Yuan Sun, Huayin Li, Jin Bai, Demin Xu, Weizhong Wu, Qian Zhu, and Mengjie Yang. Yuan Sun is affiliated with Shanghai Guanbo Law Firm. All the other members are affiliated with Zhongshan Hospital.

\section{Consent for publication}

The authors consent to publication of the research report by BMC Cancer.

\section{Competing interests}

The authors declare that they have no competing financial and non-financial interests.

\section{Author details}

'Department of General Surgery, Shanghai Public Health Clinical Center, Fudan University, Shanghai, China. ${ }^{2}$ Department of General Surgery, Zhongshan Hospital, Fudan University, Shanghai, China. ${ }^{3}$ Department of Medical Oncology, Zhongshan Hospital, Fudan University, Shanghai, China. ${ }^{4}$ GenomiCare Biotechnology (Shanghai) Co. Ltd., Shanghai, China.

Received: 2 April 2020 Accepted: 23 September 2020

Published online: 28 October 2020

References

1. Yang $Z$, et al. Incidence, distribution of histological subtypes and primary sites of soft tissue sarcoma in China. Cancer Biol Med. 2019;16:565-74.

2. Krishnan Unni CDMFK, Mertens F, Fletcher CDM, Unni FMK. Pathology and genetics of tumours of soft tissue and bone; 2002.

3. Taylor BS, et al. Advances in sarcoma genomics and new therapeutic targets. Nat Rev Cancer. 2011;11:541-57.

4. The Cancer Genome Atlas Research Network, Comprehensive and Integrated Genomic Characterization of Adult Soft Tissue Sarcomas. Cell. 2017;171:950-965 e28

5. Zang Y, et al. Comprehensive analysis of potential immunotherapy genomic biomarkers in 1000 Chinese patients with cancer. Cancer Med. 2019;8:4699-708.

6. McLaren W, et al. The Ensembl variant effect predictor. Genome Biol. 2016; $17: 122$.

7. Huber W, et al. Orchestrating high-throughput genomic analysis with bioconductor. Nat Methods. 2015;12:115-21.

8. Mayakonda A, Lin DC, Assenov Y, Plass C, Koeffler HP. Maftools: efficient and comprehensive analysis of somatic variants in cancer. Genome Res. 2018;28: 1747-56.

9. Kandoth C. vcf2maf v1.6.16. GitHub Repos. 2018; https:/doi.org/https:// github.com/mskcc/vcf2maf/releases/tag/v1.6.16.

10. Boeva $V$, et al. Control-FREEC: a tool for assessing copy number and allelic content using next-generation sequencing data. Bioinformatics. 2012;28: 423-5.

11. Mermel CH, et al. GISTIC2.0 facilitates sensitive and confident localization of the targets of focal somatic copy-number alteration in human cancers. Genome Biol. 2011;12:R41.

12. Subramanian A, et al. Gene set enrichment analysis: a knowledge-based approach for interpreting genome-wide expression profiles. Proc Natl Acad Sci U S A. 2005;102:15545-50.

13. Chalmers ZR, et al. Analysis of 100,000 human cancer genomes reveals the landscape of tumor mutational burden. Genome Med. 2017;9:34.

14. Hause RJ, Pritchard CC, Shendure J, Salipante SJ. Classification and characterization of microsatellite instability across 18 cancer types. Nat Med. 2016;22:1342-50.

15. Niu B, et al. MSIsensor: microsatellite instability detection using paired tumor-normal sequence data. Bioinformatics. 2014;30:1015-6.

16. Cieslik M, et al. The use of exome capture RNA-seq for highly degraded RNA with application to clinical cancer sequencing. Genome Res. 2015;25: 1372-81.

17. Karn T, et al. A small hypoxia signature predicted $\mathrm{pCR}$ response to Bevacizumab in the Neoadjuvant GeparQuinto breast Cancer trial. Clin Cancer Res. 2020;26:1896-904.

18. Dobin A, et al. STAR: ultrafast universal RNA-seq aligner. Bioinformatics. 2013;29:15-21.

19. Kovaka S, et al. Transcriptome assembly from long-read RNA-seq alignments with StringTie2. Genome Biol. 2019;20:278. 
20. Love MI, Huber W, Anders S. Moderated estimation of fold change and dispersion for RNA-seq data with DESeq2. Genome Biol. 2014;15:550.

21. Kanehisa M, Furumichi M, Tanabe M, Sato Y, Morishima K. KEGG: new perspectives on genomes, pathways, diseases and drugs. Nucleic Acids Res. 2017:45:D353-61.

22. Zhou G, et al. NetworkAnalyst 3.0: a visual analytics platform for comprehensive gene expression profiling and meta-analysis. Nucleic Acids Res. 2019;47:W234-41.

23. A. D. View ORCID ProfileBrian J, Stransky HN, Li B, Yang X, Tickle T, Bankapur A, Ganote C, Doak TG, Pochet N, Sun J, Wu CJ, Gingeras TR, Regev A. STARFusion: Fast and Accurate Fusion Transcript Detection from RNA-Seq. biorxiv. 2017. https://doi.org/10.1101/120295.

24. Petitprez $F$, et al. B cells are associated with survival and immunotherapy response in sarcoma. Nature. 2020;577:556-60.

25. Becht $E$, et al. Estimating the population abundance of tissue-infiltrating immune and stromal cell populations using gene expression. Genome Biol. 2016;17:218

26. Chakravarty D, et al. OncoKB: A Precision Oncology Knowledge Base. JCO Precis Oncol. 2017:1-16.

27. Cubillos-Rojas M, et al. The E3 ubiquitin protein ligase HERC2 modulates the activity of tumor protein p53 by regulating its oligomerization. J Biol Chem. 2014;289:14782-95.

28. $\mathrm{Xi} Y$, et al. HMGA2 promotes adipogenesis by activating C/EBPß-mediated expression of PPARy. Biochem Biophys Res Commun. 2016;472:617-23.

29. Zaidi MR, Okada Y, Chada KK. Misexpression of full-length HMGA2 induces benign Mesenchymal tumors in mice. Cancer Res. 2006;66:7453-9.

30. Dei Tos AP, et al. Coordinated expression and amplification of the MDM2, CDK4, and HMGl-C genes in atypical lipomatous tumours. J Pathol. 2000; 190:531-6.

31. Kadoch C, et al. Proteomic and bioinformatic analysis of mammalian SWI/ SNF complexes identifies extensive roles in human malignancy. Nat Genet. 2013:45:592-601.

32. Drost J, et al. BRD7 is a candidate tumour suppressor gene required for $\mathrm{p} 53$ function. Nat Cell Biol. 2010;12:380-9.

33. Skaaby $\mathrm{T}$, et al. Filaggrin loss-of-function mutations and incident cancer: a population-based study. Br J Dermatol. 2014;171:1407-14.

34. Coindre JM. New WHO classification of tumours of soft tissue and bone. Ann Pathol. 2012;32:S115-6.

35. Sawada R, et al. MDM2 copy number increase: a poor prognostic, molecular event in esophageal squamous cell carcinoma. Hum Pathol. 2019;89:1-9.

36. Casadei $L$, et al. MDM2 derived from dedifferentiated Liposarcoma extracellular vesicles induces MMP2 production from Preadipocytes. Cancer Res. 2019;79:4911-22.

37. Kim YJ, et al. Co-expression of MDM2 and CDK4 in transformed human mesenchymal stem cells causes high-grade sarcoma with a dedifferentiated liposarcoma-like morphology. Lab Investig. 2019;99:1309-20.

38. Mao L, et al. Melatonin suppression of aerobic glycolysis (Warburg effect), survival signalling and metastasis in human leiomyosarcoma. J Pineal Res. 2016;60:167-77.

39. Burattini $\mathrm{S}$, et al. Melatonin action in tumor skeletal muscle cells: an ultrastructural study. Acta Histochem. 2016;118:278-85.

40. Hirata $\mathrm{M}$, et al. Integrated exome and RNA sequencing of dedifferentiated liposarcoma. Nat Commun. 2019;10:5683.

41. Yang $\mathbf{W}$, et al. Immunogenic neoantigens derived from gene fusions stimulate T cell responses. Nat Med. 2019;25:767-75.

42. Tawbi HA, et al. Pembrolizumab in advanced soft-tissue sarcoma and bone sarcoma (SARC028): a multicentre, two-cohort, single-arm, open-label, phase 2 trial. Lancet Oncol. 2017;18:1493-501.

43. D'Angelo SP, et al. Nivolumab with or without ipilimumab treatment for metastatic sarcoma (Alliance A091401): two open-label, non-comparative, randomised, phase 2 trials. Lancet Oncol. 2018;19:416-26.

44. Toulmonde M, et al. Use of PD-1 targeting, macrophage infiltration, and IDO pathway activation in sarcomas: a phase 2 clinical trial. JAMA Oncol. 2018:4:93-7.

45. Chen DS, Mellman I. Elements of cancer immunity and the cancer-immune set point. Nature. 2017;541:321-30.

46. Cristescu R, et al. Pan-tumor genomic biomarkers for PD-1 checkpoint blockade-based immunotherapy. Science (80- ). 2018;362

47. Becht $E$, et al. Immune and stromal classification of colorectal Cancer is associated with molecular subtypes and relevant for precision immunotherapy. Clin Cancer Res. 2016;22:4057-66.

\section{Publisher's Note}

Springer Nature remains neutral with regard to jurisdictional claims in published maps and institutional affiliations.

\section{Ready to submit your research? Choose BMC and benefit from:}

- fast, convenient online submission

- thorough peer review by experienced researchers in your field

- rapid publication on acceptance

- support for research data, including large and complex data types

- gold Open Access which fosters wider collaboration and increased citations

- maximum visibility for your research: over $100 \mathrm{M}$ website views per year

At BMC, research is always in progress.

Learn more biomedcentral.com/submissions 